\title{
Some Derivative-Free Quadrature Rules for Numerical Approximations of Cauchy Principal Value of Integrals
}

\author{
Rabindranath Das, ${ }^{1}$ Manoj Kumar Hota, ${ }^{2}$ and Manoranjan Bej ${ }^{3}$ \\ ${ }^{1}$ Department of Mathematics \& Computer Science, G.M. Autonomous College, Sambalpur, Odisha, India \\ ${ }^{2}$ School of Applied Sciences, Department of Mathematics, KIIT University, Bhubaneswar, Odisha 751024, India \\ ${ }^{3}$ Department of Mathematics, GITA, Bhubaneswar, Odisha 752054, India
}

Correspondence should be addressed to Manoj Kumar Hota; manoj.hota1974@gmail.com

Received 11 November 2013; Accepted 10 December 2013; Published 17 March 2014

Academic Editors: F. W. S. Lima, H. J. Ruskin, and J. G. Zhou

Copyright (c) 2014 Rabindranath Das et al. This is an open access article distributed under the Creative Commons Attribution License, which permits unrestricted use, distribution, and reproduction in any medium, provided the original work is properly cited.

Some derivative-free six-point quadrature rules for approximate evaluation of Cauchy principal value of integrals have been constructed in this paper. Rules are numerically verified by suitable integrals, their degrees of precision have been determined, and their respective errors have been asymptotically estimated.

\section{Introduction}

Recently Das and Hota [1] have constructed a derivative-free 8 -point quadrature rule for numerical evaluation of complex Cauchy Principal Value of integrals of type

$$
I(f)=P \int_{z_{0}-h}^{z_{0}+h} \frac{f(z)}{z-z_{0}} d z
$$

along the directed line segment $L$, from the point $z_{0}-h$ to the point $z_{0}+h$, and $f(z)$ is assumed to be an analytic function in a domain $\Omega$ containing $L$.

The objective of this paper is to obtain some other quadrature rules having six-nodes not involving derivative of the function for numerical approximation of the complex $\mathrm{CPV}$ integrals given in (1) from the family of rules given by Das and Hota [1].

\section{Formulation of Rules}

Das and Hota [1] have given the following derivative-free 8point parametric quadrature rule of degree of precision at most ten to approximate the integrals of the type given in (1):

$$
\begin{aligned}
& R(f, \alpha) \\
& \quad=w_{0} f\left(z_{0}\right)+w_{1}\left[f\left(z_{0}+h\right)-f\left(z_{0}-h\right)\right]
\end{aligned}
$$

$$
\begin{aligned}
& +w_{2}\left[f\left(z_{0}+i h\right)-f\left(z_{0}-i h\right)\right] \\
& +w_{3}\left[f\left(z_{0}+\alpha h\right)-f\left(z_{0}-\alpha h\right)\right] \\
& +w_{4}\left[f\left(z_{0}+i \alpha h\right)-f\left(z_{0}-i \alpha h\right)\right], \quad i=\sqrt{-1},
\end{aligned}
$$

where

$$
\begin{gathered}
w_{0}=0, \quad w_{1}=\left[\frac{18-70 \alpha^{4}}{105\left(1-\alpha^{4}\right)}\right], \\
w_{2}=i\left[\frac{35 \alpha^{4}-3}{105\left(1-\alpha^{4}\right)}\right], \quad w_{3}=\left[\frac{10+42 \alpha^{2}}{105 \alpha^{3}\left(1-\alpha^{4}\right)}\right], \\
w_{4}=i\left[\frac{10-42 \alpha^{2}}{105 \alpha^{3}\left(1-\alpha^{4}\right)}\right],
\end{gathered}
$$

the rule given in (2) is of precision eight for $\alpha \in(0,1)$.

However, the rule $R(f, \alpha)$ may be reduced to a six point rule for suitable values of the parameter " $\alpha$." without altering 
its algebraic degree of precision that is eight. These rules are as follows:

(i) $\alpha=\sqrt[4]{9 / 35}$;

for this value of $\alpha$; the weight $w_{1}$ in (3) is zero and the rule given in (2) becomes a six point rule denoted by $R_{1}(f)$ given as:

$$
\begin{aligned}
& R_{1}(f)=\frac{i}{13}\left[f\left(z_{0}+i h\right)-f\left(z_{0}-i h\right)\right]+\frac{\sqrt[4]{35}[5 \sqrt{35}+63]}{117 \sqrt{3}} \\
& \times\left\{f\left(z_{0}+\sqrt[4]{\frac{9}{35}} h\right)-f\left(z_{0}-\sqrt[4]{\frac{9}{35}} h\right)\right\} \\
& +i \frac{\sqrt[4]{35}[5 \sqrt{35}-63]}{117 \sqrt{3}} \\
& \times\left\{f\left(z_{0}+i \sqrt[4]{\frac{9}{35}} h\right)-f\left(z_{0}-i \sqrt[4]{\frac{9}{35} h}\right)\right\} .
\end{aligned}
$$

(ii) $\alpha=\sqrt{5 / 21}$;

in this case the weight $w_{4}=0$ and the corresponding rule (denoted by $R_{2}(f)$ ) is:

$$
\begin{aligned}
R_{2}(f)= & \frac{221}{1560}\left[f\left(z_{0}+h\right)-f\left(z_{0}-h\right)\right] \\
& -\frac{2 i}{195}\left[f\left(z_{0}+i h\right)-f\left(z_{0}-i h\right)\right] \\
& +\frac{441}{520} \times \sqrt{\frac{21}{5}}\left[\begin{array}{c}
f\left(z_{0}+\sqrt{\frac{5}{21}} h\right) \\
-f\left(z_{0}-\sqrt{\frac{5}{21}} h\right)
\end{array}\right]
\end{aligned}
$$

(iii) $\alpha=\sqrt[4]{3 / 35}$

as in the case of two cases noted above, we found here that the weight $w_{2}=0$ for this value of $\alpha$; and the rule denoted by $R_{3}(f)$ becomes:

$$
\begin{aligned}
R_{3}(f)= & \frac{1}{8}\left[f\left(z_{0}+h\right)-f\left(z_{0}-h\right)\right] \\
& +\frac{5(35)^{3 / 4}+21 \sqrt{3} \times \sqrt[4]{35}}{48 \times \sqrt[4]{27}} \\
& \times\left\{f\left(z_{0}+\sqrt[4]{\frac{3}{35} h}\right)-f\left(z_{0}-\sqrt[4]{\frac{3}{35}} h\right)\right\} \\
& +i \frac{5(35)^{3 / 4}-21 \sqrt{3} \times \sqrt[4]{35}}{48 \times \sqrt[4]{27}} \\
& \times\left\{f\left(z_{0}+i \sqrt[4]{\frac{3}{35}} h\right)-f\left(z_{0}-i \sqrt[4]{\frac{3}{35}} h\right)\right\} .
\end{aligned}
$$

Each of these rules, that is, $R_{2}(f)$, and $R_{3}(f)$ is a six-point rule. For the numerical integration of the integral (1) it is required to evaluate the function $f(z)$ at six points instead of eight points as in the case, the rule proposed by Das and Hota [1]. Both the rules $R_{2}(f)$ and $R_{3}(f)$ have $z_{0} \pm h$ as nodes and hence they are closed type of rules.

It is pertinent to note here that the degree of precision of each of the rules $R_{1}(f), R_{2}(f)$, and $R_{3}(f)$ is eight which is the same as that of the rule $R(f, \alpha)$ except for the value of $\alpha=1 / \sqrt{3}$ in which case it becomes a rule of precision ten; however, in this case, evaluation of function at 8 nodes is required in approximation of integrals.

\section{Error Analysis}

The error $E(f, \alpha)$ associated with the rule $R(f, \alpha)$ as given in (2) is

$$
E(f, \alpha)=I(f)-R(f, \alpha)
$$

We assume here that the function $f(z)$ is analytic in the disc

$$
\Omega=\left\{z \in C:\left|z-z_{0}\right| \leq \rho=r|h| ; r>1\right\} .
$$

Under this assumption, $f(z)$ can be expanded in terms of the Taylor's series about the point $z=z_{0}$ in the $\operatorname{disc} \Omega$ as

$$
f(z)=\sum_{n=0}^{\infty} a_{n}\left(z-z_{0}\right)^{n}
$$

where $a_{n}=f^{(n)}\left(z_{0}\right) /(n)$ ! are the Taylor's coefficients.

As the series given in (9) is uniformly convergent in $\Omega$, we obtain by integrating both sides of the series (9) term by term and obtain

$$
\begin{aligned}
I(f)= & 2 h f^{\prime}\left(z_{0}\right)+\frac{2 h^{3}}{3(3 !)} f^{\prime \prime}\left(z_{0}\right)+\frac{2 h^{5}}{5(5 !)} f^{(5)}\left(z_{0}\right) \\
& +\frac{2 h^{7}}{7(7 !)} f^{(7)}\left(z_{0}\right)+\frac{2 h^{9}}{9(9 !)} f^{(9)}\left(z_{0}\right) \\
& +\frac{2 h^{11}}{11(11 !)} f^{(11)}\left(z_{0}\right)+\cdots
\end{aligned}
$$

Again by expanding each term of the rule $R(f, \alpha)$ given in (2) by Taylor's series expansion about the point $z=z_{0}$ in the disc $\Omega$ and then after simplification we obtain

$$
\begin{aligned}
R(f, \alpha)= & 2 h f^{\prime}\left(z_{0}\right)+\frac{2 h^{3}}{3(3 !)} f^{\prime \prime}\left(z_{0}\right)+\frac{2 h^{5}}{5(5 !)} f^{(5)}\left(z_{0}\right) \\
& +\frac{2 h^{7}}{7(7 !)} f^{(7)}\left(z_{0}\right)+\frac{2 h^{9}}{(9 !)}\left[\frac{1-5 \alpha^{4}+4 \alpha^{8}}{5\left(1-\alpha^{4}\right)}\right] f^{(9)}\left(z_{0}\right) \\
& +\frac{2 h^{11}}{(11 !)}\left[\frac{3-7 \alpha^{4}+4 \alpha^{8}}{21\left(1-\alpha^{4}\right)}\right] f^{(11)}\left(z_{0}\right)+\cdots .
\end{aligned}
$$


Further, by substituting $\alpha=\sqrt[4]{3 / 35}, \alpha=\sqrt{5 / 21}$, and $\alpha=$ $\sqrt[4]{9 / 35}$ in the expression given in (11) we have

$$
\begin{array}{rl}
R_{1}(f)=2 & h f^{\prime}\left(z_{0}\right)+\frac{2 h^{3}}{3(3 !)} f^{\prime \prime}\left(z_{0}\right) \\
& +\frac{2 h^{5}}{5(5 !)} f^{(5)}\left(z_{0}\right)+\frac{2 h^{7}}{7(7 !)} f^{(7)}\left(z_{0}\right) \\
& -\frac{206 h^{9}}{2275(9 !)} f^{(9)}\left(z_{0}\right)+\frac{46 h^{11}}{245(11 !)} f^{(11)}\left(z_{0}\right)+\cdots
\end{array}
$$$$
R_{2}(f)=2 h f^{\prime}\left(z_{0}\right)+\frac{2 h^{3}}{3(3 !)} f^{\prime \prime}\left(z_{0}\right)
$$$$
+\frac{2 h^{5}}{5(5 !)} f^{(5)}\left(z_{0}\right)+\frac{2 h^{7}}{7(7 !)} f^{(7)}\left(z_{0}\right)
$$$$
+\frac{68 h^{9}}{2205(9 !)} f^{(9)}\left(z_{0}\right)+\frac{2446 h^{11}}{2205(11 !)} f^{(11)}\left(z_{0}\right)+\cdots,
$$

$$
\begin{array}{rl}
R_{3}(f)=2 & h f^{\prime}\left(z_{0}\right)+\frac{2 h^{3}}{3(3 !)} f^{\prime \prime}\left(z_{0}\right) \\
& +\frac{2 h^{5}}{5(5 !)} f^{(5)}\left(z_{0}\right)+\frac{2 h^{7}}{7(7 !)} f^{(7)}\left(z_{0}\right) \\
& +\frac{46 h^{9}}{175(9 !)} f^{(9)}\left(z_{0}\right)+\frac{62 h^{11}}{245(11 !)} f^{(11)}\left(z_{0}\right)+\cdots
\end{array}
$$

respectively.

Denoting

$$
E_{k}(f)=I(f)-R_{k}(f), \quad \forall k=1,2,3,
$$

and then making the appropriate substitutions for $I(f)$ and $R_{k}(f)$ in (15) we obtain after simplification the following expressions for $E_{k}(f), k=1,2,3$ :

$$
\begin{aligned}
& E_{k}(f) \\
& = \begin{cases}(0.23) \frac{h^{9}}{9 !} f^{(9)}\left(z_{0}\right)+\frac{16}{2695} \frac{h^{11}}{(11 !)} f^{(11)}\left(z_{0}\right)+\cdots & \text { for } k=1 \\
(-0.08) \frac{h^{9}}{9 !} f^{(9)}\left(z_{0}\right)-\frac{8384}{101871} \frac{h^{11}}{(11 !)} f^{(11)}\left(z_{0}\right)+\cdots & \text { for } k=2 \\
(-0.04) \frac{h^{9}}{9 !} f^{(9)}\left(z_{0}\right)-\frac{192}{2695} \frac{h^{11}}{(11 !)} f^{(11)}\left(z_{0}\right)+\cdots & \text { for } k=3,\end{cases}
\end{aligned}
$$

which in turn imply

$$
\left|E_{k}(f)\right| \approx O\left(|h|^{9}\right), \quad \forall k=1,2,3 .
$$

From the first term of the error expression given in (16), it is also evident that amongst the three rules $\left(R_{1}(f), R_{2}(f)\right.$, and $\left.R_{3}(f)\right), R_{3}(f)$ shall integrate more accurately than the other two rules $R_{1}(f), R_{2}(f)$. Further, in the approximation of the integral by rules $R_{1}(f)$ and $R_{2}(f)$, the rule $R_{2}(f)$ is better than the rule $R_{1}(f)$ in the sense that the approximation obtained by $R_{2}(f)$ is closer to the true value compared to that obtained by $R_{1}(f)$.This observation is also substantiated by the numerical results obtained in the approximation of some standard integrals by these rules given in Section 4 .

3.1. Error Bounds. The error bounds of the two quadrature rules $R_{2}(f)$ and $R_{3}(f)$ constructed in this paper have been obtained here by following the technique due to Lether [3]. Since the derivation of error bound is similar to each of the two rules, we have derived the error bound of the rule $R_{3}(f)$ only and it is given in Theorem 1 , and the error bound of the rule $R_{2}(f)$ is only stated in Theorem 2 following Theorem 1 .

It is noted here that the error bound of the quadrature rule $R_{1}(f)$ given in (4) cannot be determined in the same way as it is done for other two cases, that is, $R_{2}(f)$ and $R_{3}(f)$, by following the technique due to Lether [3] for the reasons explained below.

Since $E_{1}(f)$ denotes the truncation error in approximation of integral $I(f)$ by the rule $R_{1}(f)$,

$$
I(f)=R_{1}(f)+E_{1}(f)
$$

and $E_{1}$ being a linear operator, we obtain from (9) the following:

$$
E_{1}(f)=\sum_{\mu=4}^{\infty} a_{2 \mu+1} h^{2 \mu+1} E_{1}\left(t^{2 \mu+1}\right)
$$

by using the transformation $z=z_{0}+h t, t \in[-1,1]$, and from this we get

$$
E_{1}(f)=\sum_{\mu=4}^{\infty} 2 a_{2 \mu+1} h^{2 \mu+1} \chi(\mu),
$$

where

$$
\begin{aligned}
& \chi(\mu) \\
& =\left[\frac{\frac{1}{2 \mu+1}+(-1)^{\mu}\left\{\frac{1}{13}+\frac{\sqrt[4]{35}(5 * \sqrt{35}-63)}{117 \sqrt{3}} *\left(\frac{9}{35}\right)^{((2 \mu+1) / 4)}\right\}}{-\frac{\sqrt[4]{35}(5 * \sqrt{35}+63)}{117 \sqrt{3}} *\left(\frac{9}{35}\right)^{((2 \mu+1) / 4)}}\right]
\end{aligned}
$$

which is not of one sign for $\mu \geq 3$.

However, its asymptotic error estimates have been given in (17). Next we consider:

\subsection{Error Bound of the Rule $R_{3}(f)$}

Theorem 1. If $f(z)$ is analytic in a closed disc

$$
\Omega=\left\{z \in C:\left|z-z_{0}\right| \leq \rho=r|h| ; r>1\right\},
$$

then

$$
\left|E_{3}(f)\right| \leq 2 M e_{3}(r),
$$


where

$$
\begin{aligned}
& e_{3}(r) \\
& =\left|\ln \left(\frac{r+1}{r-1}\right)-\left\{\frac{210 r^{5}-140 r^{3}-46}{105 r^{6}-105 r^{4}-9 r^{2}+9}\right\}\right|,
\end{aligned}
$$

which $\rightarrow 0$ for $r \rightarrow \infty$.

Proof. Here

$$
\begin{array}{r}
E_{3}\left[\left(z-z_{0}\right)^{k}\right] \\
=\int_{z_{0}-h}^{z_{0}+h} \frac{\left(z-z_{0}\right)^{k}}{z-z_{0}} d z-R_{3}\left[\left(z-z_{0}\right)^{k}\right]=0, \\
\text { for } k=0 \text { (1) } 8 .
\end{array}
$$

Again since $E_{3}(f)$ denotes the truncation error in approximation of integral $I(f)$ by the rule $R_{3}(f)$, that is,

$$
I(f)=R_{3}(f)+E_{3}(f),
$$

and $E_{3}$ being a linear operator, we obtain from (9) the following:

$$
E_{3}(f)=\sum_{\mu=4}^{\infty} a_{2 \mu+1} h^{2 \mu+1} E_{3}\left(t^{2 \mu+1}\right)
$$

by using the transformation $z=z_{0}+h t, t \in[-1,1]$, and from this we get

$$
E_{3}(f)=\sum_{\mu=4}^{\infty} 2 a_{2 \mu+1} h^{2 \mu+1} \varphi(\mu),
$$

where

$$
\begin{gathered}
\varphi(\mu)=\left[\frac{2}{2 \mu+1}-\left\{\frac{1}{4}+2 *\left(\frac{3}{35}\right)^{((2 \mu+1) / 4)}\right.\right. \\
\left.\left.*\left(A-(-1)^{\mu} * B\right)\right\}\right], \\
A=\left(\frac{35}{3}\right)^{1 / 4} *\left(\frac{5 \sqrt{35}+21 \sqrt{3}}{48 \sqrt{3}}\right), \\
B=\left(\frac{35}{3}\right)^{1 / 4} *\left(\frac{5 \sqrt{35}-21 \sqrt{3}}{48 \sqrt{3}}\right) .
\end{gathered}
$$

Now

$$
\begin{aligned}
& \varphi(\mu) \\
& = \begin{cases}\frac{2}{2 \mu+1}-\left[\frac{1}{4}+\frac{7}{8} *\left(\frac{3}{35}\right)^{\mu / 2}\right] ; & \text { for } \mu \text { as even } \\
\frac{2}{2 \mu+1}-\left[\frac{1}{4}+\frac{5}{12} *\left(\frac{3}{35}\right)^{(\mu-1) / 2}\right] ; & \text { for } \mu \text { as odd }\end{cases}
\end{aligned}
$$

which implies

$$
\varphi(\mu)<\frac{2}{2 \mu+1}-\frac{1}{4}<0, \quad \text { for } \mu \geq 4 .
$$

Now by Cauchy-inequality [4],

$$
\begin{aligned}
\left|E_{3}(f)\right| & \leq 2 M \sum_{\mu=4}^{\infty} \frac{1}{r^{2 \mu+1}}\left|E_{3}\left(t^{2 \mu+1}\right)\right| \\
& =2 M e_{3}(r)
\end{aligned}
$$

where

$$
\begin{aligned}
M= & \operatorname{Max}_{|z|=\rho}|f(z)|, \\
e_{3}(r)= & \left|E\left[\left(1-\frac{t}{r}\right)^{-1}\right]\right| \\
= & \mid \ln \left(\frac{r+1}{r-1}\right) \\
& -\left\{\frac{210 r^{5}-140 r^{3}-46}{105 r^{6}-105 r^{4}-9 r^{2}+9}\right\} \mid,
\end{aligned}
$$

which $\rightarrow 0$ as $r \rightarrow \infty$. This completes the proof of the theorem.

It will not be out of place to mention here that the quantity $e_{3}(r)$ is defined as error constant associated with a quadrature rule by Lether [5]. It is tacitly assumed that the function is an entire function so that the error-constant approaches to zero as $r$ approaches to infinite. In fact, we observe that $e_{3}(r)$ is practically zero for $r \geq 9$ as it is seen from the Table 18 .

Hence, for all practical purposes we find that the function need to be analytic in a disc $\Omega$ of finite radius $r$.

Theorem 2. If $f(z)$ is analytic in a closed disc

$$
\Omega=\left\{z \in C:\left|z-z_{0}\right| \leq \rho=r|h| ; r>1\right\},
$$

then

$$
\left|E_{2}(f)\right| \leq 2 M e_{2}(r),
$$

where

$$
\begin{aligned}
e_{2}(r)=\mid \ln \left(\frac{r+1}{r-1}\right) \\
-\left\{\frac{32760 r^{5}+3120 r^{3}-28808 r}{16380 r^{6}-3900 r^{4}-16380 r^{2}+3900}\right\} \mid,
\end{aligned}
$$

which $\rightarrow 0$ for $r \rightarrow \infty$.

This corollary can also be established in the same vain as it is done in Theorem 1 since

$$
E_{2}(f)=\sum_{\mu=4}^{\infty} 2 a_{2 \mu+1} h^{2 \mu+1} \psi(\mu),
$$


where

$$
\begin{gathered}
\psi(\mu) \\
=\left\{\begin{aligned}
\frac{2}{2 \mu+1}-\left[\frac{237}{780}+\frac{441}{260} *\left(\frac{5}{21}\right)^{\mu}\right] ; & \text { for } \mu \text { as even } \\
\frac{2}{2 \mu+1}-\left[\frac{205}{780}+\frac{441}{260} *\left(\frac{5}{21}\right)^{\mu}\right] ; & \text { for } \mu \text { as odd }, \\
\psi(\mu)<0, & \text { for } \mu \geq 4 .
\end{aligned}\right.
\end{gathered}
$$

The error constants $e_{k}(r), k=2,3$, have been numerically evaluated for values of $(r>1)$ and presented in Table 18 in the concluding part of this paper. A close look at the table reveals that the rule $R_{3}(f)$ more accurately integrates a complex Cauchy principal value of integrals numerically than the rule $R_{2}(f)$. This assertion is evident from the numerical values of the error constants $e_{r}^{s}$ given in Table 18 and from their respective graphs drawn together in Figure 1 at the end of this paper. It is observed that

$$
e_{3}(r) \leq e_{2}(r),
$$

for any fixed but arbitrary value of $(r>1)$.

Further, from the asymptotic error estimates of the rules $R_{1}(f), R_{2}(f)$, and $R_{3}(f)$ constructed in this paper for the numerical evaluation of complex CPV integrals, it also follows that

$$
\left|E_{3}(f)\right| \leq\left|E_{2}(f)\right| \leq\left|E_{1}(f)\right| .
$$

This observation is in fact very much noticeable from the numerical integration of the integral that we have taken and the results of numerical integration presented in tables given in Section 4.

\section{Numerical Verification}

4.1. Approximate Evaluation of Complex Cauchy Principal Value of Integrals. The integrals considered here are

$$
\begin{gathered}
I_{1}=P \int_{-i}^{i} \frac{(1+z) e^{z}}{z} d z, \\
I_{2}=P \int_{-i}^{i} \frac{(1+z \cos z)}{z} d z, \\
I_{3}=P \int_{(1+i) / 4}^{(-1+i) / 4} \frac{\tan ^{-1} z}{z} d z, \\
I_{4}=P \int_{(1+i) / 2}^{3(1+i) / 2} \frac{\sin z}{z-(1+i)} d z, \\
I_{5}=P \int_{-i}^{i} \frac{e^{z}}{z} d z .
\end{gathered}
$$

Results of numerical integration of the above five CPV

\begin{tabular}{|c|c|c|}
\hline \multirow{2}{*}{ Rules } & \multicolumn{2}{|l|}{ Integral } \\
\hline & $\begin{array}{c}\text { Approximate value of } \\
I_{3}=P \int_{(1+i) / 4}^{(-1+i)}\left(\tan ^{-1} z / z\right) d z\end{array}$ & Absolute error \\
\hline$R_{1}(f)$ & $-0.506612+0.492763 * i$ & $2.2 \times 10^{-6}$ \\
\hline$R_{2}(f)$ & $-0.506614+0.492765 * i$ & $1.0 \times 10^{-6}$ \\
\hline$R_{3}(f)$ & $-0.506614+0.492765 * i$ & $1.0 \times 10^{-6}$ \\
\hline Exact value & $-0.506614+0.492764 * i$ & $* *$ \\
\hline
\end{tabular}
integrals are given in Tables 1, 2, 3, 4, and 5 .

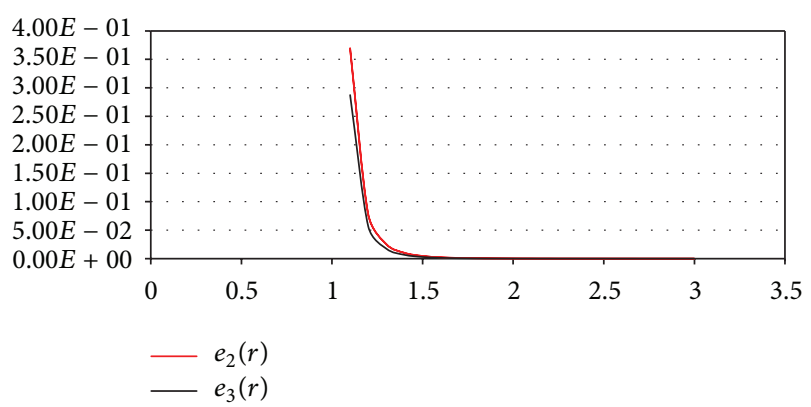

Figure 1

TABLE 1

\begin{tabular}{lcc}
\hline Rules & \multicolumn{2}{c}{ Integral } \\
& $\begin{array}{c}\text { Approximate value of } \\
I_{1}=\end{array} \int_{-i}^{i}\left((1+z) e^{z} / z\right) d z$ & Absolute error \\
\hline$R_{1}(f)$ & $3.575102 * i$ & $6.0 \times 10^{-6}$ \\
$R_{2}(f)$ & $3.575111 * i$ & $3.0 \times 10^{-6}$ \\
$R_{3}(f)$ & $3.575109 * i$ & $1.0 \times 10^{-6}$ \\
\hline Exact value & $3.575108 * i$ & $* *$ \\
\hline
\end{tabular}

TABLE 2

\begin{tabular}{lcc}
\hline Rules & \multicolumn{2}{c}{ Integral } \\
& $\begin{array}{c}\text { Approximate value of } \\
I_{2}=P \int_{-i}^{i}((1+z \cos z) / z) d z\end{array}$ & Absolute error \\
& $2.350397 * i$ & $5.0 \times 10^{-6}$ \\
$R_{1}(f)$ & $2.350405 * i$ & $3.0 \times 10^{-6}$ \\
$R_{2}(f)$ & $2.350403 * i$ & $1 \times 10^{-6}$ \\
$R_{3}(f)$ & $2.350402 * i$ & $* *$ \\
\hline Exact value & & \\
\hline
\end{tabular}

TABLE 3

TABLE 4

\begin{tabular}{lcc}
\hline Rules & \multicolumn{2}{c}{$\begin{array}{c}\text { Integral } \\
\text { Approximate value of } \\
\end{array}$} \\
& $P \int_{4}^{3(1+i) / 2}(\sin z /(z-(1+i))) d z$ & Absolute error \\
\hline$R_{1}(f)$ & $1.8175586-0.2057251 * i$ & $1 \times 10^{-7}$ \\
$R_{2}(f)$ & $1.8175587-0.2057251 * i$ & 0.0 \\
$R_{3}(f)$ & $1.8175587-0.2057251 * i$ & 0.0 \\
\hline Exact value & $1.8175587-0.2057251 * i$ & $* *$ \\
\hline
\end{tabular}


TABLE 5

\begin{tabular}{lcc}
\hline Rules & \multicolumn{2}{c}{ Integral } \\
& $\begin{array}{c}\text { Approximate value of } \\
I_{5}=P \int_{-i}^{i}\left(e^{z} / z\right) d z\end{array}$ & Absolute error \\
\hline$R_{1}(f)$ & $1.8921655 * i$ & $6 \times 10^{-7}$ \\
$R_{2}(f)$ & $1.8921664 * i$ & $2.9 \times 10^{-7}$ \\
$R_{3}(f)$ & $1.8921663 * i$ & $1.9 \times 10^{-7}$ \\
\hline Exact value & $1.8921661 * i$ & $* *$ \\
\hline
\end{tabular}

TABLE 6

\begin{tabular}{lcc}
\hline Rules & \multicolumn{2}{c}{ Integral } \\
& $\begin{array}{c}\text { Approximate value of } \\
J_{1}=\int_{-i}^{i} e^{z} d z\end{array}$ & Absolute error \\
\hline$R_{1}(f)$ & $1.682936 * i$ & $5.9 \times 10^{-6}$ \\
$R_{2}(f)$ & $1.682944 * i$ & $2.0 \times 10^{-6}$ \\
$R_{3}(f)$ & $1.682943 * i$ & $1 \times 10^{-6}$ \\
\hline Exact value & $1.682942 * i$ & $* *$ \\
\hline
\end{tabular}

TABLE 7

\begin{tabular}{lcc}
\hline Rules & \multicolumn{2}{c}{ Integral } \\
& $\begin{array}{c}\text { Approximate value of } \\
J_{2}=\int_{-i / 2}^{i / 2} \cos z d z\end{array}$ & Absolute error \\
\hline$R_{1}(f)$ & $1.042190600 * i$ & $1.1 \times 10^{-8}$ \\
$R_{2}(f)$ & $1.042190615 * i$ & $4.0 \times 10^{-9}$ \\
$R_{3}(f)$ & $1.042190613 * i$ & $2.0 \times 10^{-9}$ \\
\hline Exact value & $1.042190611 * i$ & $* *$ \\
\hline
\end{tabular}

TABLE 8

\begin{tabular}{lcc}
\hline Rules & \multicolumn{2}{c}{ Integral } \\
& $\begin{array}{c}\text { Approximate value of } \\
J_{3}=\int_{-(1+i) / \sqrt{2}}^{(1+i) / \sqrt{2}} z e^{z} d z\end{array}$ & Absolute error \\
\hline$R_{1}(f)$ & $-0.516863+0.422579 * i$ & $4.7 \times 10^{-5}$ \\
$R_{2}(f)$ & $-0.516823+0.422624 * i$ & $1.4 \times 10^{-5}$ \\
$R_{3}(f)$ & $-0.516830+0.422618 * i$ & $6.0 \times 10^{-6}$ \\
\hline Exact value & $-0.516830+0.422612 * i$ & $* *$ \\
\hline
\end{tabular}

TABLE 9

\begin{tabular}{lcc}
\hline Rules & \multicolumn{2}{c}{$\begin{array}{c}\text { Integral } \\
\end{array}$} \\
& $\begin{array}{c}\text { Approximate value of } \\
Q_{1}=P \int_{-1}^{1}\left(e^{x} / x\right) d x\end{array}$ & Absolute error \\
\hline$R_{1}(f)$ & 2.11450111 & $6.4 \times 10^{-7}$ \\
$R_{2}(f)$ & 2.11450199 & $2.4 \times 10^{-7}$ \\
$R_{3}(f)$ & 2.11450186 & $1 \times 10^{-7}$ \\
\hline Exact value & 2.11450175 (Longman $[2])$ & $* *$ \\
\hline
\end{tabular}

TABLE 10

\begin{tabular}{lcc}
\hline Rules & \multicolumn{2}{c}{ Integral } \\
& $Q_{2}=P \int_{-1 / 4}^{1 / 4}\left((x+1) / x\left(x^{2}+1\right)\right) d x$ & Absolute error \\
\hline$R_{1}(f)$ & 0.4899564 & $9.0 \times 10^{-7}$ \\
$R_{2}(f)$ & 0.4899576 & $3.0 \times 10^{-7}$ \\
$R_{3}(f)$ & 0.4899574 & $1.0 \times 10^{-7}$ \\
\hline Exact value & 0.4899573 & $* *$ \\
\hline
\end{tabular}

TABLE 11

\begin{tabular}{lcc}
\hline Rules & \multicolumn{2}{c}{$\begin{array}{c}\text { Integral } \\
\end{array}$} \\
& $\begin{array}{c}\text { Approximate value of } \\
Q_{3}=P \int_{-1 / 2}^{1 / 2} d x / x(x+2)\end{array}$ & Absolute error \\
\hline$R_{1}(f)$ & -0.2554123 & $5.0 \times 10^{-7}$ \\
$R_{2}(f)$ & -0.2554130 & $2.0 \times 10^{-7}$ \\
$R_{3}(f)$ & -0.2554129 & $1.0 \times 10^{-7}$ \\
\hline Exact value & -0.2554128 & $* *$ \\
\hline
\end{tabular}

TABLE 12

\begin{tabular}{lcc}
\hline Rules & \multicolumn{2}{c}{$\begin{array}{c}\text { Integral } \\
\end{array}$} \\
& $\begin{array}{c}\text { Approximate value of } \\
Q_{4}=P \int_{-1}^{1}\left((x+1) e^{x} / x\right) d x\end{array}$ & Absolute error \\
\hline$R_{1}(f)$ & 4.464898 & $6.0 \times 10^{-6}$ \\
$R_{2}(f)$ & 4.464907 & $3.0 \times 10^{-6}$ \\
$R_{3}(f)$ & 4.464905 & $1.0 \times 10^{-6}$ \\
\hline Exact value & 4.464904 & $* *$ \\
\hline
\end{tabular}

TABLE 13

\begin{tabular}{lcc}
\hline Rules & \multicolumn{2}{c}{$\begin{array}{c}\text { Integral } \\
\end{array}$} \\
& $\begin{array}{c}\text { Approximate value of } \\
T_{1}=\int_{-1}^{1} e^{x} d x\end{array}$ & Absolute error \\
\hline$R_{1}(f)$ & 2.3503966 & $5.8 \times 10^{-6}$ \\
$R_{2}(f)$ & 2.3504046 & $2.2 \times 10^{-6}$ \\
$R_{3}(f)$ & 2.3504034 & $1.0 \times 10^{-8}$ \\
\hline Exact value & Correct to seven decimal places & $* *$ \\
\hline
\end{tabular}

4.2. Approximations of Integrals of Analytic Functions over a Line Segment in the Complex Plane. In this subsection of numerical experimentation, quadrature rules $R_{1}(f), R_{2}(f)$ and $R_{3}(f)$ constructed in this paper have been employed for approximate evaluation of integrals of analytic functions in complex variable over a line segment $L$ joining the points 
TABLE 14

\begin{tabular}{lcc}
\hline Rules & \multicolumn{2}{c}{ Integral } \\
& $\begin{array}{c}\text { Approximate value of } \\
T_{2}=\int_{0}^{1} e^{-x^{2}} d x\end{array}$ & Absolute error \\
\hline$R_{1}(f)$ & 0.746832 & $8.0 \times 10^{-6}$ \\
$R_{2}(f)$ & 0.746821 & $3.0 \times 10^{-6}$ \\
$R_{3}(f)$ & 0.746823 & $1.0 \times 10^{-6}$ \\
\hline \multirow{2}{*}{ Exact value } & 0.746824 & $* *$ \\
\hline
\end{tabular}

TABLE 15

\begin{tabular}{lcc}
\hline Rules & \multicolumn{2}{c}{ Integral } \\
& $\begin{array}{c}\text { Approximate value of } \\
T_{3}=\int_{0}^{1} e^{x^{2}} d x\end{array}$ & Absolute error \\
\hline$R_{1}(f)$ & 1.46256 & $9.0 \times 10^{-5}$ \\
$R_{2}(f)$ & 1.46268 & $3.0 \times 10^{-5}$ \\
$R_{3}(f)$ & 1.46266 & $1.0 \times 10^{-5}$ \\
\hline Exact value & 1.46265 & $* *$ \\
\hline
\end{tabular}

\section{TABLE 16}

\begin{tabular}{lcc}
\hline Rules & \multicolumn{2}{c}{$\begin{array}{c}\text { Integral } \\
\text { Approximate value of } \\
T_{4}=\int_{1}^{3}\left(\sin ^{2} x / x\right) d x\end{array}$} \\
\hline$R_{1}(f)$ & 0.79486 & Absolute error \\
$R_{2}(f)$ & 0.79480 & $4.0 \times 10^{-5}$ \\
$R_{3}(f)$ & 0.79481 & $2.0 \times 10^{-5}$ \\
\hline Exact value & 0.79482 & $1.0 \times 10^{-5}$ \\
\hline
\end{tabular}

TABLE 17

\begin{tabular}{lcc}
\hline Rules & \multicolumn{2}{c}{ Integral } \\
& $\begin{array}{c}\text { Approximate value of } \\
T_{5}=\int_{0}^{1}\left(1 /\left(1+e^{x}\right)\right) d x\end{array}$ & Absolute error \\
\hline$R_{1}(f)$ & 0.37988552 & $3.0 \times 10^{-8}$ \\
$R_{2}(f)$ & 0.37988548 & $1.0 \times 10^{-8}$ \\
$R_{3}(f)$ & 0.37988549 & 0.0 \\
\hline Exact value & 0.37988549 & $* *$ \\
\hline
\end{tabular}

$z_{0}-h$ to $z_{0}+h$ in the complex plane $C$. The integrals considered here are

$$
\begin{gathered}
J_{1}=\int_{-i}^{i} e^{z} d z, \quad J_{2}=\int_{-i / 2}^{i / 2} \cos z d z \\
J_{3}=\int_{-(1+i) / \sqrt{2}}^{(1+i) / \sqrt{2}} z e^{z} d z .
\end{gathered}
$$

Results of numerical integration of the above integrals are given in Tables 6,7 , and 8 .
TABLE 18

\begin{tabular}{lcc}
\hline$r$ & $e_{2}(r)$ & $e_{3}(r)$ \\
\hline 1.1 & 0.3695183127 & 0.2873549877 \\
2.1 & 0.0001455392 & 0.0000800958 \\
3.1 & 0.0000036852 & 0.0000018628 \\
4.1 & 0.0000002826 & 0.0000001382 \\
5.1 & 0.0000000388 & 0.0000000187 \\
6.1 & 0.0000000076 & 0.0000000036 \\
7.1 & 0.0000000019 & 0.0000000009 \\
8.1 & 0.0000000006 & 0.0000000002 \\
9.1 & 0.0000000002 & 0.0000000000 \\
\hline
\end{tabular}

4.3. Evaluation of Some Real Cauchy Principal Value of Integrals. In this subsection of numerical verification, the quadrature rules $R_{1}(f), R_{2}(f)$, and $R_{3}(f)$ as formulated in this paper for numerical integration of complex Cauchy principal value integrals have been applied for the approximate evaluation of the following real Cauchy principal value of integrals. The results of approximations are depicted in Tables $9,10,11$, and 12 , respectively. The integrals considered here are

$$
\begin{gathered}
Q_{1}=P \int_{-1}^{1} \frac{e^{x}}{x} d x, \\
Q_{2}=P \int_{-1 / 4}^{1 / 4} \frac{x+1}{x\left(x^{2}+1\right)} d x, \\
Q_{3}=P \int_{-1 / 2}^{1 / 2} \frac{d x}{x(x+2)}, \\
Q_{4}=P \int_{-1}^{1} \frac{(x+1) e^{x}}{x} d x .
\end{gathered}
$$

4.4. Approximate Evaluation of Real Definite Integrals. In this subsection of numerical experimentation, quadrature rules $R_{1}(f), R_{2}(f)$, and $R_{3}(f)$ constructed in this paper have been successfully employed for approximate evaluation of real definite integrals without having any kind of singularities. The rules have been practically verified with the help of the following integrals:

$$
\begin{gathered}
T_{1}=\int_{-1}^{1} e^{x} d x, \quad T_{2}=\int_{0}^{1} e^{-x^{2}} d x \\
T_{3}=\int_{0}^{1} e^{x^{2}} d x, \quad T_{4}=\int_{1}^{3} \frac{\sin ^{2} x}{x} d x \\
T_{5}=\int_{0}^{1} \frac{1}{1+e^{x}} d x
\end{gathered}
$$

Results of numerical integration of the above five integrals are given in Tables 13, 14, 15, 16, and 17.

\section{Conclusion}

The rules constructed in this paper successfully integrate to at least six decimal places of the complex Cauchy principal 
value integrals we have numerically evaluated in this paper. Each of the rules does not require evaluation of the function at $z=z_{0}$; the point of singularity. Also, it is not required to evaluate derivative of the integrand at any of its nodes, which is a positive advantage over the existing rules found in the literature. [6-8].

Again, these rules can also be applied for numerical integration of:

(i) Complex definite integrals on the line segment having end points $z_{0}-h$ and $z_{0}+h$ in the complex plane $C$.

(ii) These rules one can apply for the numerical integration of real definite integrals, and

(iii) It is also shown that the rules constructed numerically integrate real Cauchy principal value integrals to at least six decimal places accurately.

The numerical values of the error constants $e_{2}(r)$ and $e_{3}(r)$ associated with the quadrature rules $R_{2}(f)$ and $R_{3}(f)$ meant for numerical evaluation of the complex Cauchy principal value of integrals as given (5) and (6), respectively, in this paper are depicted in Table 18.

From this table it is quite apparent that the error constant $e_{3}(r)$ corresponding to the rule $R_{3}(f)$ moves faster towards zero than that of the rules $R_{2}(f)$ although both the rules are six-point rule with the same degree of precision. This observation is substantiated by the results obtained in the approximation of the integrals given in Section 4 .

It is worth mentioning here that three mixed quadrature rules can be constructed following the technique suggested by Das and Pradhan [6]. The degree of precision of each of these mixed quadrature rules will be 10 . The authors are investigating two parameter rules of the same problem, that is, approximation of integral given in (1).

\section{Conflict of Interests}

The authors declare that they have done this research work on their own interest. No organization/institution has given financial assistance to carry this research work. The authors declare that they have no conflict of interests. Also, this paper has not been published in any other journal.

\section{References}

[1] R. N. Das and M. K. Hota, "A derivative free quadrature rule for numerical approx. of complex Cauchy principal value integrals," Applied Mathematical Sciences, vol. 6, no. 111, pp. 5533-5540, 2012.

[2] I. M. Longman, "On numerical evaluation of Cauchy principal values of integrals," MTAC, vol. 12, pp. 205-207, 1958.

[3] F. G. Lether, "Error bound for fully symmetric cubature rules," SIAM Journal on Numerical Analysis, vol. 8, no. 1, pp. 49-60, 1971.

[4] J. B. Conway, Functions of One Complex Variable, Narosa, New York, NY, USA, 2nd edition, 1980.

[5] B. P. Acharya and T. Mahapatra, "Numerical computation of complex Cauchy principal value integrals," International Journal of Computer Mathematics, vol. 43, no. 3-4, pp. 147-151, 1992.
[6] R. N. Das and G. Pradhan, "A modification of Simpson's (1/3) ${ }^{r d}$ rule," International Journal of Mathematical Education in Science and Technology, vol. 28, no. 6, pp. 908-910, 1997.

[7] M. M. Chawla and N. Jayarajan, "Quadrature formulas for cauchy principal value integrals," Computing, vol. 15, no. 4, pp. 347355, 1975.

[8] P. J. Davis and P. Robinowitz, Methods of Numerical Integration, Academic Press, New York, NY, USA, 2nd edition, 1975. 


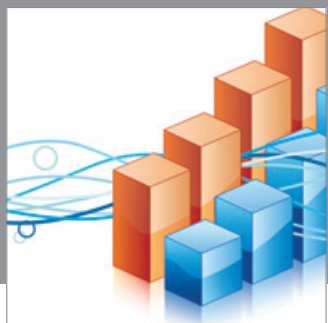

Advances in

Operations Research

mansans

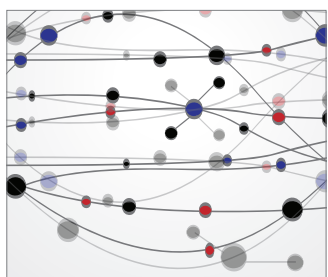

The Scientific World Journal
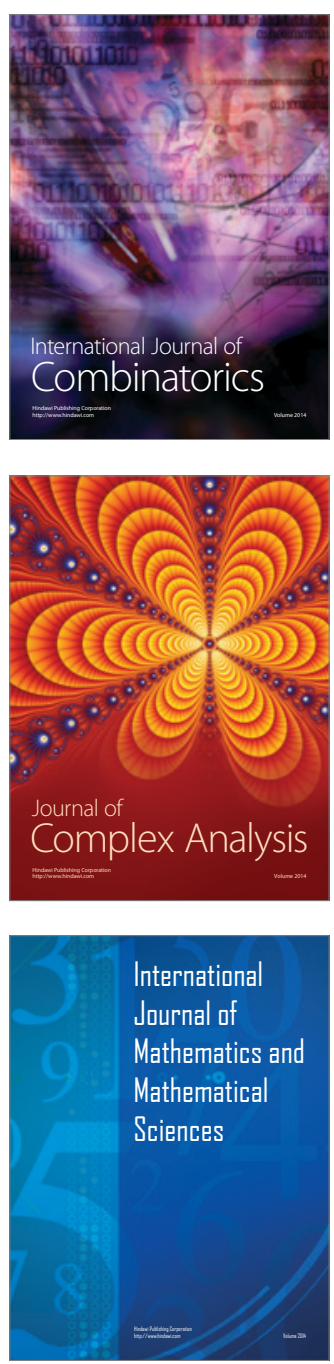
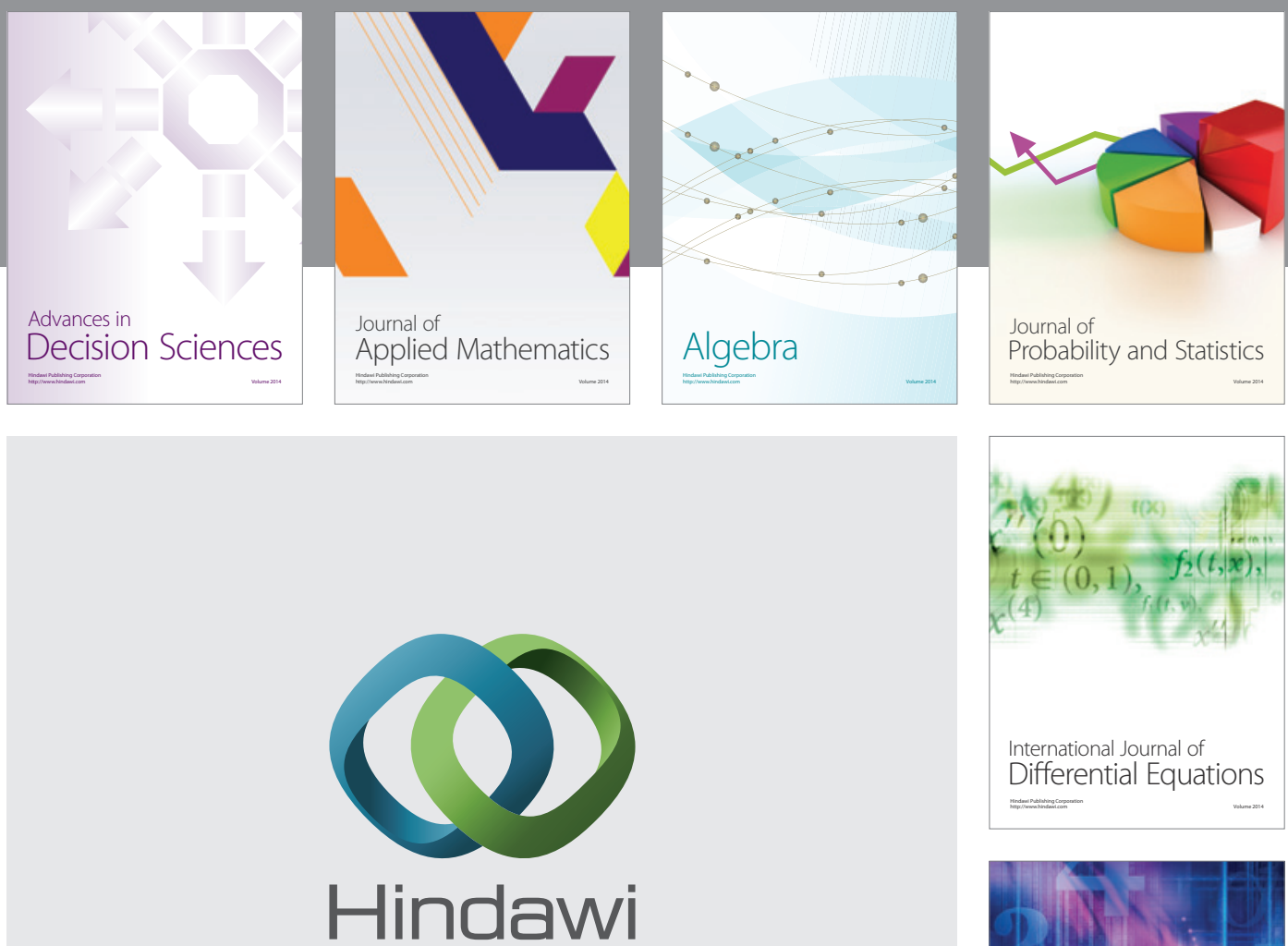

Submit your manuscripts at http://www.hindawi.com
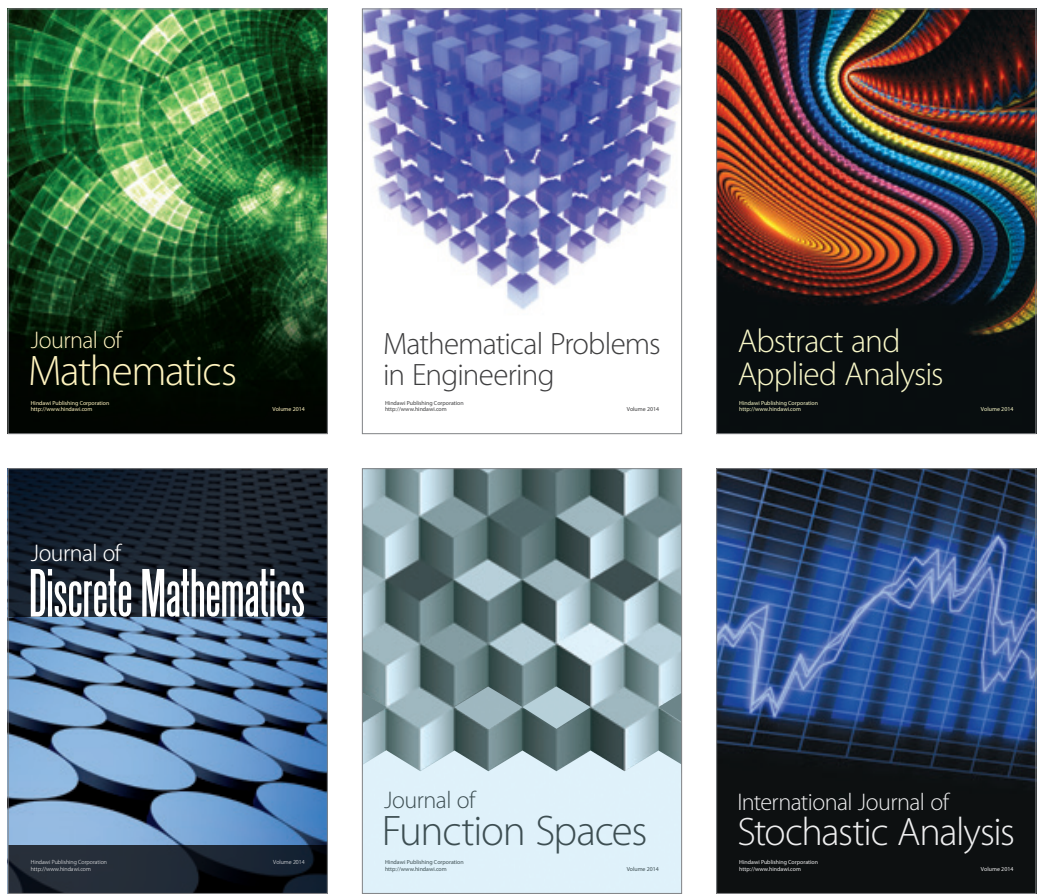

Journal of

Function Spaces

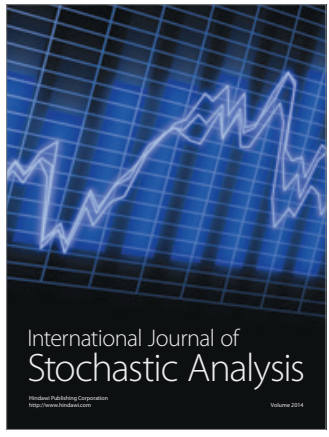

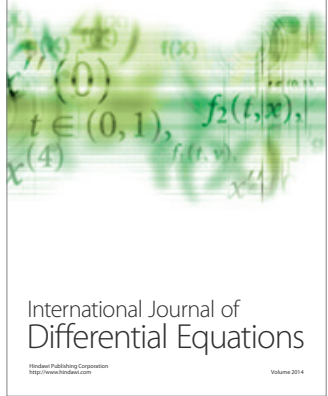
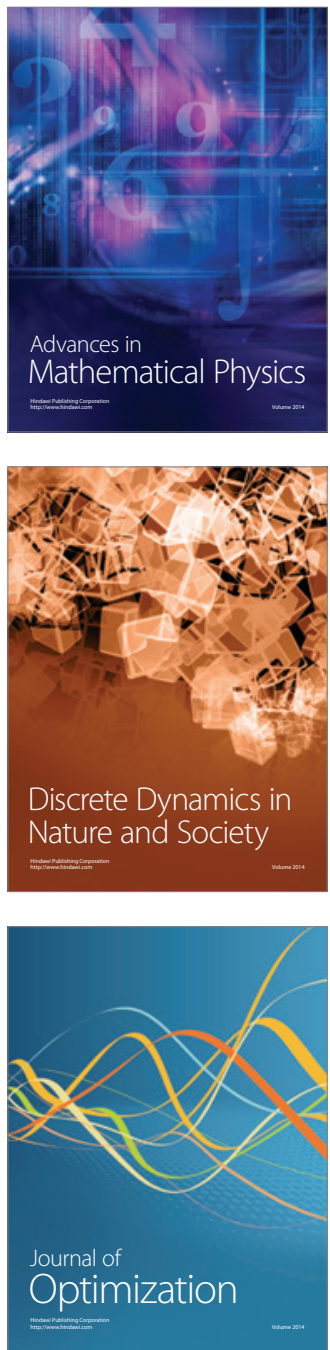